\title{
ECOLOGIE, BIOLOGIE \\ ET CYCLE DE REPRODUCTION DES GAMMARES DU 'ASSI (= ORONTE, LIBAN) [CRUSTACES, AMPHIPODES]
}

par N. J. ALOUF 1

Trois espèces de Gammares apparentés au groupe pulex, G. syriacus, G. laticoxalis et $G$. oronticus, vivent en sympatrie dans la partie amont de l'Oronte. La reproduction de $G$. oronticus est continue durant toute l'année, un ralentissement relatif s'observe au printemps. Le sex-ratio est en faveur des mâles dans une station, il est à égalité dans une autre et nettement en faveur des femelles dans les deux autres. Les pourcentages de femelles gravides par rapport au total de femelles et au total des Gammares sont en corrélation.

\section{Ecology, biology and reproductive cycle of Gammarus from the River 'Assi (= Oronte, Lebanon) (Crustacea Amphipoda).}

Three species of gammarids related to the pulex-group, G. syriacus, G. laticoxalis and $G$. oronticus, are sympatric in the upper part of the river Oronte. The reproductive cycle of $G$. oronticus shows that the reproduction is continuous with a relative resting period in spring. The sex-ratio is $1: 1$ at only one station, there are more males than females at another one and more females than males at two others. The percentage of mature females and the sex-ratio are correlated.

\section{1. - INTRODUCTION}

Nous avons trouvé que l'activité sexuelle des Gammares colonisant la vallée du Litani dépend du régime des précipitations atmosphériques (Alouf 1979 a). Cette activité est continue pendant toute l'année, bien que la saison des pluies ne s'étende que sur sept mois. Le pourcentage minimal de femelles gravides ne descend pas, dans les conditions normales, au-dessous de $60 \%$ à la fin de l'été, après quatre ou cinq mois de sécheresse; le maximum est supérieur à $90 \%$, à la fin de l'automne et en hiver. La densité et la taille moyenne des femelles évoluent en corrélation avec l'activité sexuelle. Le sex-ratio varie d'une station à une autre.

Les conditions écologiques prévalant dans la vallée de l'Oronte, que ce soit la température de l'eau, le régime des pluies ou la photopé-

1. Faculté des Sciences, Université Libanaise, Hadath-Beyrouth, Liban. Travail subventionné par le Conseil National de la Recherche Scientifique au Liban. 
riode, sont assez semblables à celles de la vallée du Litani, bien que la région soit à climat subdésertique. Doit-on s'attendre à ce que l'évolution de l'activité sexuelle, du sex-ratio, de la taille moyenne et de la densité suive les mêmes modalités dans les deux bassins?

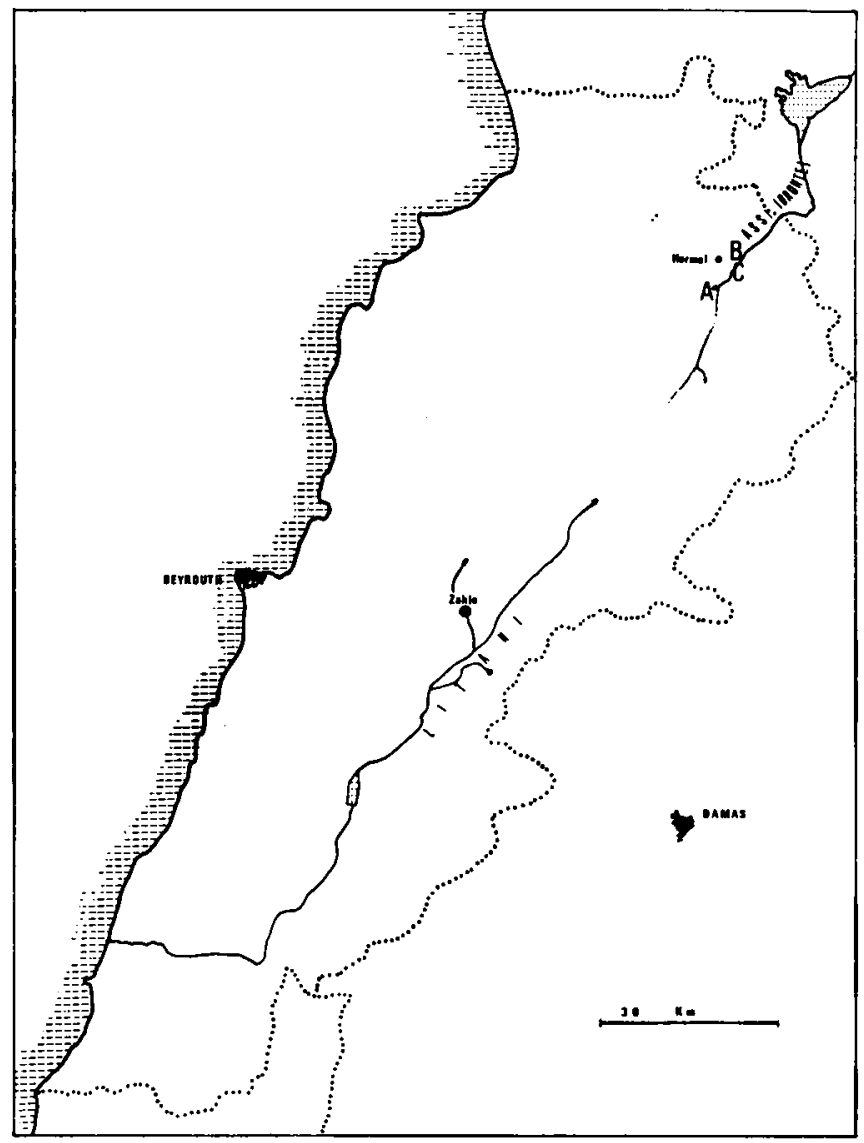

Fig. 1. - Carte de la région étudiée.

Berg (1948) remarqua que, dans les pays nordiques, la reproduction et la taille moyenne de $G$. pulex augmentaient au printemps et diminuaient au début de l'automne. Hynes, pour sa part (1955), observa que, dans les Iles Britanniques, la taille moyenne était maximale en hiver et minimale en été et que le pourcentage de femelles sexuellement mûres et le sex-ratio (pris dans le sens défini plus bas) augmentaient et diminuaient en même temps. Les variations du sex-ratio seraient dues, selon ce dernier auteur, soit à ce que la durée de vie des mâles est plus courte que celle des femelles, soit à ce que la température détermine le sexe. Y a-t-il des analogies entre les Gammares de ces régions et ceux de l'Oronte? 


\section{2. - MATERIEL ET METHODES}

Trois espèces colonisent la partie supérieure du cours de l'Oronte (Alouf 1979 b), Gammarus syriacus Chevreux, 1895, Gammarus laticoxalis Karaman \& Pinkster, 1977 et Gammarus oronticus Alouf, 1979.

Des prélèvements, entre octobre 1974 et août 1975, ont été effectués pendant la quatrième semaine de chaque mois dans les stations suivantes :

A. La grande exsurgence de Nabh-l'Assi.

B. Une source à une dizaine de mètres du cours du fleuve.

C1. Le fleuve Oronte près du bord.

C2. Le fleuve Oronte près du milieu du cours.

Le moyen de récolte est un filet de Surber de $0,1 \mathrm{~m}^{2}$ de surface et de 3 mailles environ par $\mathrm{mm}$. Les échantillons sont pris entre $10 \mathrm{~h}$ et midi. L'analyse s'effectue au laboratoire en vue de trier les Gammares en :

- a : femelles possédant des soies aux oostégites; elles sont considérées comme sexuellement mûres, qu'elles soient gravides ou non.

$-q \mathrm{r}$ : femelles de taille supérieure à la plus petite femelle gravide et n'ayant pas de soies aux oostégites, quelle que soit la grandeur de ces derniers.

- $\hat{o}$ : mâles de taille supérieure à la taille des femelles gravides.

Les Gammares ont été mesurés de la base de l'antenne I jusqu'au telson et sont séparés en classes de $1 \mathrm{~mm}$. Les Gammares de petite taille sont considérés comme jeunes sans distinction de sexe. Ces données permettent d'étudier :

1. Le sex-ratio $q \mathrm{a} \times 100 /$ total des adultes;

2. Le pourcentage de femelles sexuellement mûres par rapport au total des femelles;

3. L'évolution de la taille;

4. La proportion des espèces vivant en sympatrie.

\section{3. - RESULTATS}

\section{1. - Station A}

La grande exsurgence de Nabh-l-'Assi, appelée aussi 'Ayn-z-Zarqa (la source ou l'œil bleu) est une très grande source dont le débit varie 
entre $11 \pm 5 \mathrm{~m}^{3} / \mathrm{s}$ en automne et $15 \pm 5 \mathrm{~m}^{3} / \mathrm{s}$ au printemps et au début de l'été (fig. 9). La température oscille entre $12,8^{\circ} \mathrm{C}$ en avril et $15^{\circ} \mathrm{C}$ en octobre. Les eaux de l'exsurgence Labouah arrivent, à ce niveau, sous la forme d'un mince filet d'eau, presque à sec en été. Le milieu présente de grandes variations durant l'année, dues aux différences de débit, ce qui se répercute sur le peuplement en Gammares. Ainsi, la faiblesse du débit en janvier 1975 nous a permis d'élever une digue; les eaux calmes, en amont, hébergeaient exclusivement Gammarus syriacus; les eaux rapides, à l'aval, étaient colonisées exclusivement par Gammarus oronticus; les deux points de prélèvements étaient distants de quelques mètres.

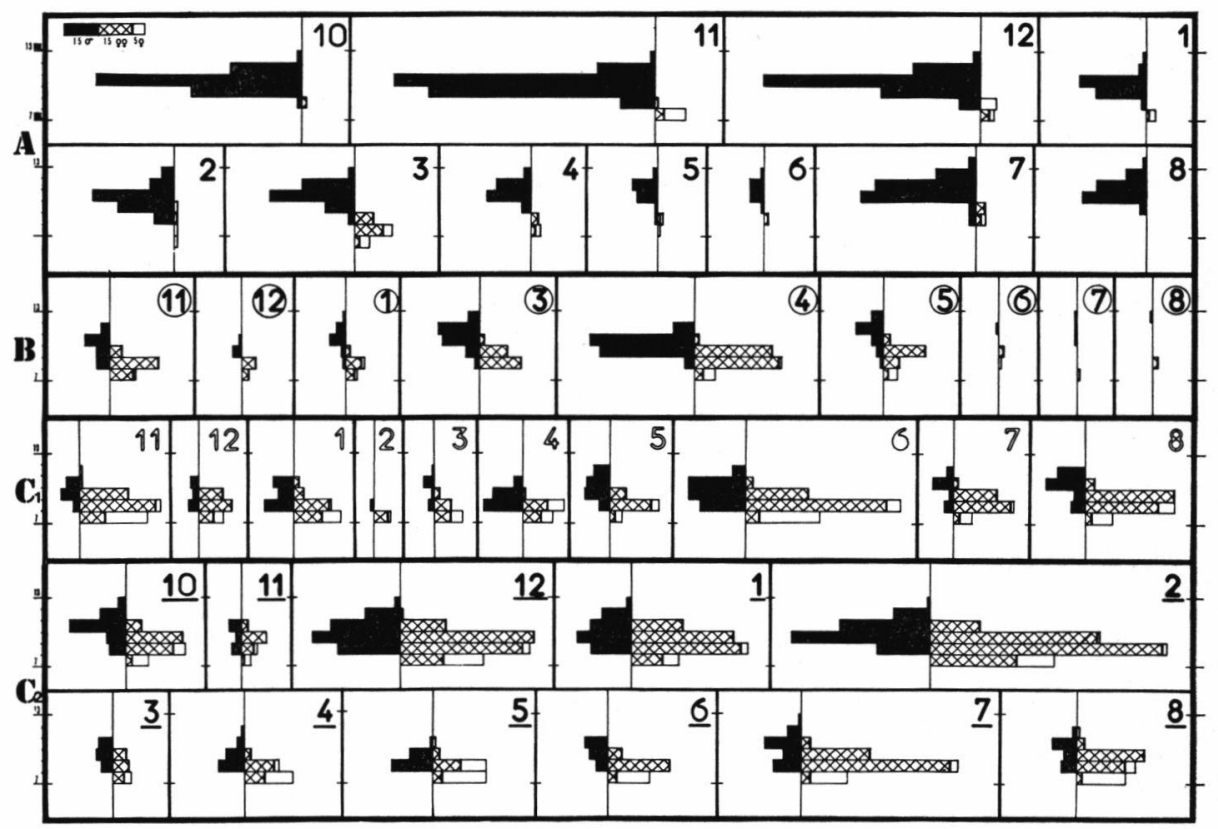

FIG. 2. - Composition mensuelle de la population de G. oronticus dans les stations $\mathrm{A}, \mathrm{B}, \mathrm{C}_{1}$ et $\mathrm{C}_{2}$. Nombre des mâles (noir), des femelles possédant des soies aux oostégites (hachures) et des femelles dépourvues de soies aux oostégites et de taille supérieure à la plus petite femelle gravide (rectangle blanc). En ordonnée, taille en $\mathrm{mm}$.

Les trois espèces G. oronticus, G. laticoxalis et G. syriacus se rencontrent dans cette station en proportions très inégales. G. oronticus est de loin la forme la plus abondante, avec 1143 individus sur un total de 1214 individus récoltés sur 11 mois et 13 prélèvements (fig. $2 \mathrm{~A})$.

Le taux de femelles gravides n'a pas été étudié chez G. oronticus, car seules 51 ㅇ a et 37 \% ont été récoltées pendant toute l’année. Le 

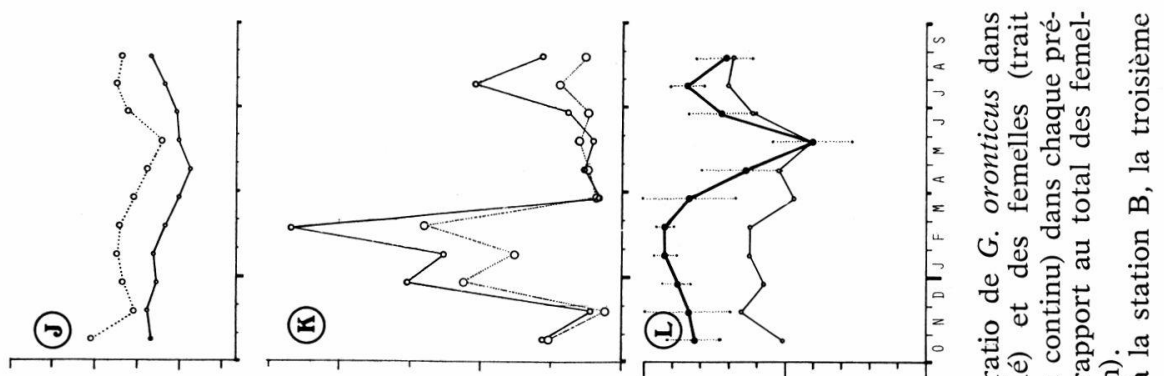

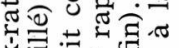

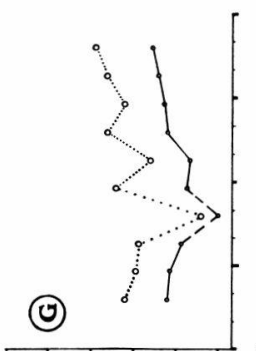

(I)

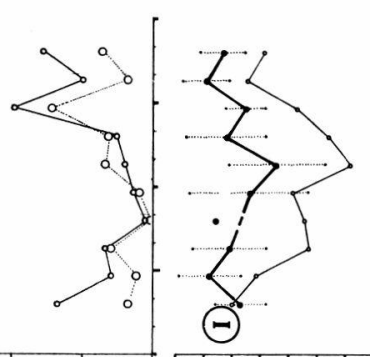

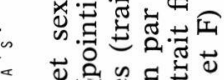

s 0

.

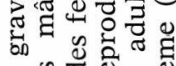

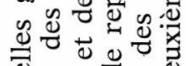

过

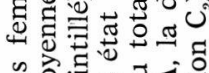

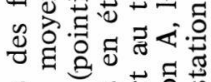

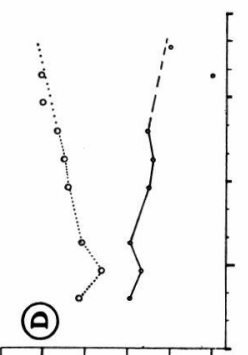

(4)

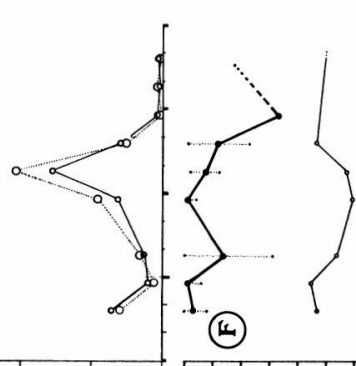

प

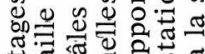

क्ष

8ิ

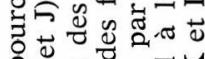

\& ov

- 00 战

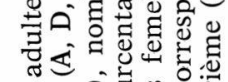

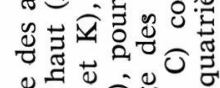

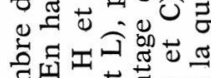

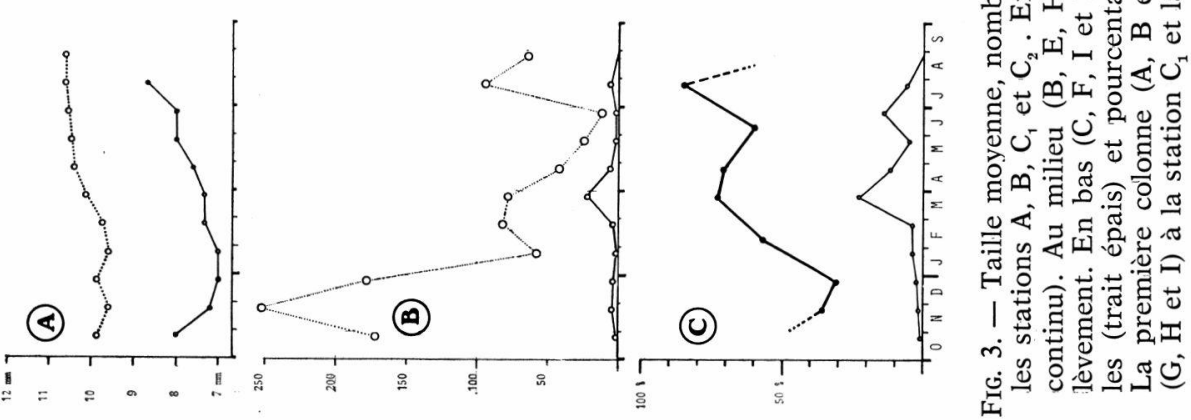


sex-ratio, nul en été, en automne et au début de l'hiver, atteignait 22 en mars. La taille moyenne des 1055 ô était $10 \mathrm{~mm}$, celle des femelles 7,5 mm (fig. $3 \mathrm{~A}, \mathrm{~B}, \mathrm{C}$ ).

Une ㅇ a, 5 ㅇ $\mathrm{r}$ et 30 ô appartenant à $G$. syriacus, ont été récoltés en janvier et 2 q a en juillet. 2 o a et 3 ô dont l'appartenance à $G$. syriacus n'est pas sûre, ont été capturés en avril, juin et juillet. Le déséquilibre numérique entre les deux sexes est semblable à ce qu'on observe chez G. oronticus. G. laticoxalis fut rencontré à la fin de l'hiver, au début du printemps et en été. Le total était de 15 \% a, 4 \% r et 9 ô.

$\mathrm{Vu}$ le nombre restreint de femelles capturées dans cette station, il n'est pas possible d'émettre une hypothèse sur les périodes d'activité ou de repos sexuel.

\section{2. - Station B}

Petite source, proche du cours de l'Oronte, elle donne naissance à un ruisselet de 1,5 $\mathrm{m}$ de largeur. La température de l'eau varie entre $12{ }^{\circ} \mathrm{C}$ en décembre et $14^{\circ} \mathrm{C}$ en août. L'eau et les pierres sont fréquemment remuées, surtout en été. Cette station héberge les trois formes $G$. laticoxalis, $G$. oronticus et $G$. syriacus dans les proportions globales

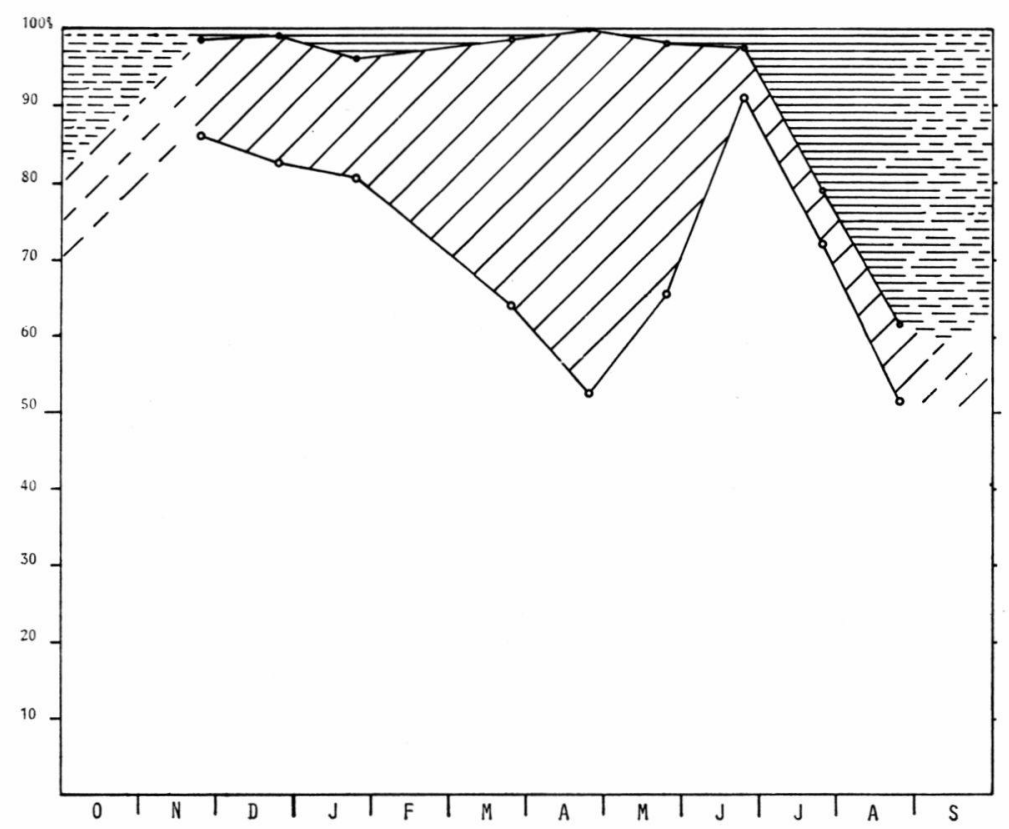

Fig. 4. - Pourcentages mensuels des trois espèces dans la station B. De bas en haut, G. laticoxalis (en blanc), G. oronticus (hachures obliques) et G. syriacus (hachures horizontales). 

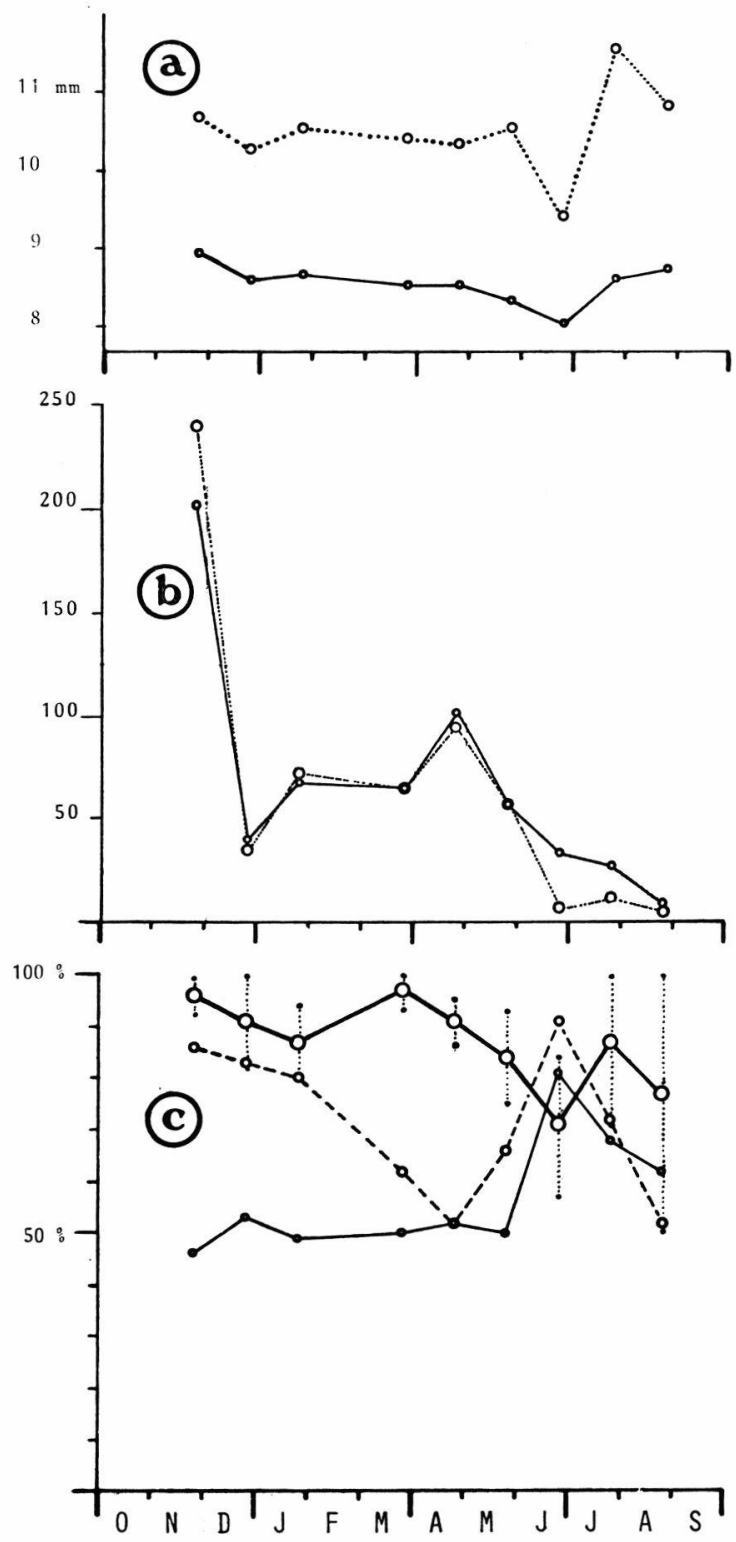

Fig. 5. - Taille moyenne, nombre des adultes, pourcentage des femelles gravides, sex-ratio et abondance relative de G. laticoxalis dans la station B; a (en haut), taille moyenne des mâles (pointillé) et des femelles (trait continu); b (au milieu), nombre des mâles (pointillé) et des femelles (trait continu) dans chaque prélèvement); c (en bas), pourcentage des femelles en état de reproduction (trait épais), pourcentage des femelles par rapport au total des adultes (trait fin) et pourcentage de $G$. laticoxalis par rapport au total des Gammares (tirets). 
de $71,7 \%, 25,8 \%$ et $2,5 \%$ respectivement, calculées sur 1761 individus récoltés en 9 prélèvements répartis sur 10 mois. Le nombre d'individus capturés en automne et en hiver s'élève à 809 , les trois formes sont dans des proportions oscillant autour de $84 \%, 14 \%$ et $2 \%$. Ces proportions deviennent $58 \%, 41 \%$ et $1 \%$ au printemps, avec 979 individus. Les pourcentages des récoltes de l'été, -157 individus seulement -, sont de $75 \%, 7 \%$ et $18 \%$ (fig. 4).

Le pourcentage mensuel de $G$. laticoxalis, espèce dominante dans cette station, passe par un premier maximum de $86 \%$ en novembre et par un autre de $91 \%$ en juin. Un premier minimum de $52,3 \%$ s'observe en avril et un second de $51,6 \%$, en août, se prolonge probablement jusqu'en septembre. Le pourcentage de $G$. oronticus passe par un seul maximum de $47,7 \%$ en novembre et par un seul minimum en été. L'abondance relative de G. syriacus est maximale, $38,5 \%$, à la fin de l'été et minimale, moins de $2 \%$, depuis l'automne jusqu'au début de l'été.

L'activité sexuelle de G. laticoxalis est continue. Le taux des femelles gravides subit des variations et passe de $96 \%$ en novembre à $87 \%$ en janvier et de $97 \%$ en mars à $71 \%$ en juin. La reprise de juillet, $87,5 \%$ est suivie d'une nouvelle chute en août, $77 \%$; ce dernier chiffre, toutefois, est basé sur 13 femelles seulement (fig. $5 \mathrm{c}$ ).

Le sex-ratio total est de 51 , les valeurs des mois d'automne, d'hiver et de printemps varient très peu autour de cette moyenne, les mois d'été sont caractérisés par une prédominance des femelles avec un maximum de 81 en juin. La taille moyenne des mâles est de $10,5 \pm 0,2 \mathrm{~mm}$, celle des femelles $8,64 \pm 0,17 \mathrm{~mm}$; la moyenne maximale, de $8,9 \mathrm{~mm}$, est observée en novembre et la minimale, de $8 \mathrm{~mm}$, en juin. L'activité sexuelle et la taille moyenne des femelles évoluent parallèlement. Il est toutefois nécessaire de noter que lorsque le sexratio et la proportion de G. laticoxalis, vis-à-vis des autres Gammares, sont au maximum, en juin ( 82 et $91 \%$ ), le taux de femelles gravides et leur taille moyenne sont au minimum $(71 \%$ et $8 \mathrm{~mm}$, voir fig. $5 \mathrm{a}, \mathrm{b}$, c). Ces données, obtenues à partir de $607 \% \mathrm{a}, 67 \% \mathrm{r}$ et 591 ô (fig. 6), montrent que les facteurs contrôlant la biologie de cette espèce dans cette station, en la favorisant au détriment des autres, sont multiples et leurs modes d'action restent à élucider.

L'activité sexuelle de $G$. oronticus est continue, le taux de femelles gravides est élevé et supérieur à $86 \%$ l'année durant. Le sex-ratio total est de 46 , les valeurs mensuelles sont inférieures à cette moyenne de janvier jusqu'en avril, de minimum de 40 s'observe en mars et le maximum de 54 en novembre-décembre. La taille moyenne, calculée sur un total de 234 of et 202 q a (fig. $2 \mathrm{~B}$ ), est de $10,4 \pm 0,13 \mathrm{~mm}$ pour les mâles et de $8,6 \pm 0,09 \mathrm{~mm}$ pour les femelles; elle est, pour ces dernières, de $8,8 \mathrm{~mm}$ durant les mois pluvieux et de $8,5 \mathrm{~mm}$ au printemps (fig. $3 \mathrm{D}, \mathrm{E}, \mathrm{F}$ ). Notons que lorsque l'abondance relative de $G$. oronti- 


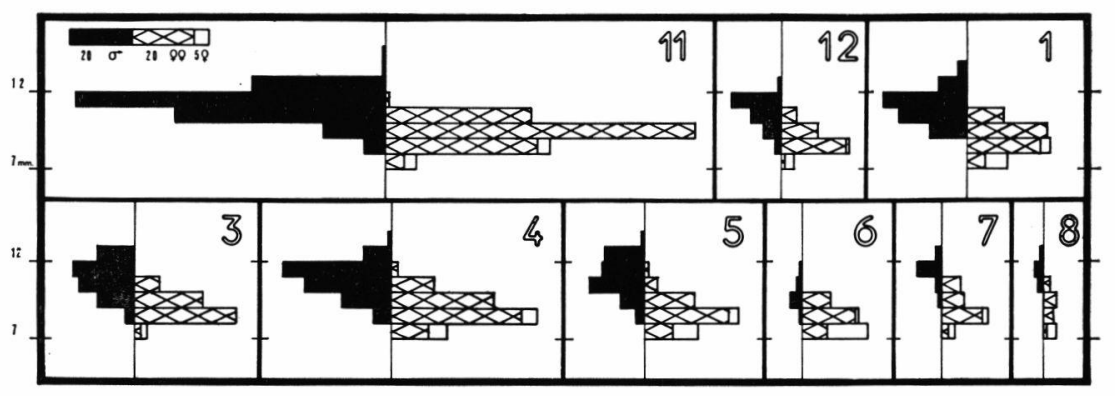

Fig. 6. - Composition mensuelle de la population de G. laticoxalis dans la station B. Nombre des mâles (noir), des femelles possédant des soies aux oostégites (hachures) et des femelles dépourvues de soies aux oostégites et de taille supérieure à la plus petite femelle gravide (rectangle blanc). En ordonnée, taille en $\mathrm{mm}$.

cus est maximale, en avril, le sex-ratio est minimal ce qui laisse supposer que les mâles sont plus nombreux à envahir la station lorsque les conditions deviennent favorables ( $f$ ig. 7).

G. syriacus n'a été récolté qu'à 48 exemplaires. La taille moyenne des mâles est de 15,9 $\pm 0,6 \mathrm{~mm}$, celle des femelles $12,2 \pm 0,5 \mathrm{~mm}$. Le sex-ratio total est de 35,5 . Huit o a, 2 o $\mathrm{r}$ et 14 fo furent récoltés de

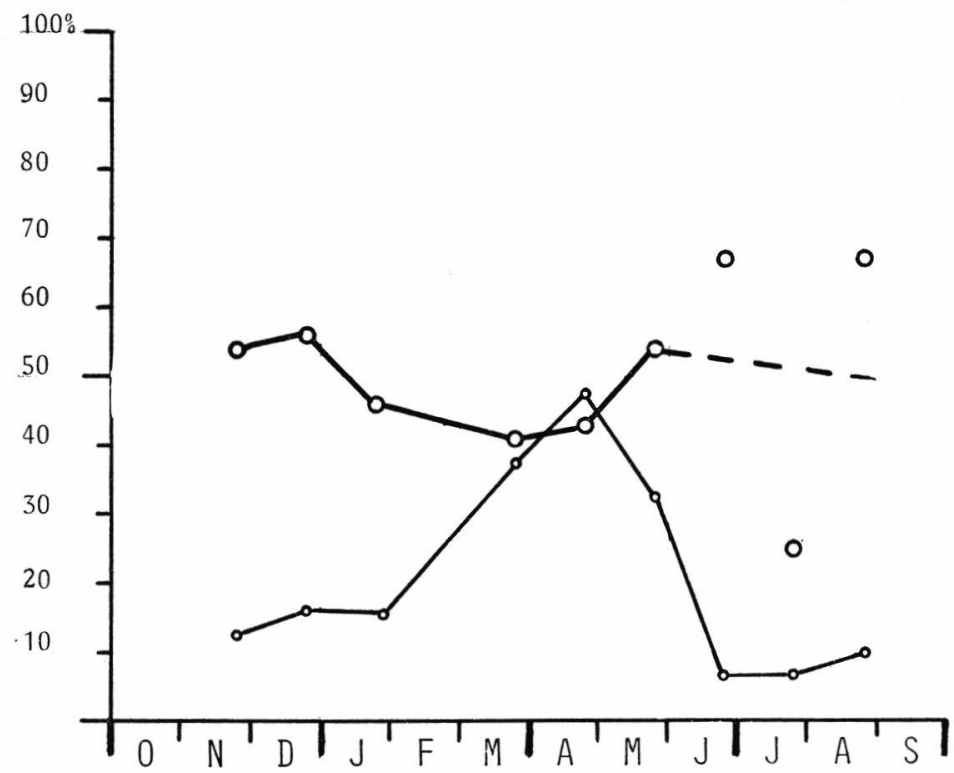

FIG. 7. - Evolution du sex-ratio et de l'abondance relative de G. oronticus dans la station B. Ligne épaisse, pourcentage des femelles par rapport au total des adultes. Ligne fine, pourcentage de G. oronticus par rapport au total des Gammares. 
novembre jusqu'en juin. Les récoltes de juillet et d'août ont donné 8 \& a, 4 \% et 2 ô. Bien que le nombre de Gammares ne soit pas important, l'échantillon est assez valable vu qu'il a été obtenu à partir de récoltes s'échelonnant sur 10 mois.

\section{3. - Station C1}

Fleuve Oronte (Nahr-l-'Assi) à $6,5 \mathrm{~km}$ de la source, là où la route menant à la localité de Hermel longe le cours du fleuve (altitude $600 \mathrm{~m}$ ). La largeur du lit oscille autour de $10 \mathrm{~m}$. Le lieu du prélèvement est à $1 \mathrm{~m}$ du bord, en eau rapide et profonde de 20 à $30 \mathrm{~cm}$. Dix prélèvements ont été effectués entre le mois de novembre 1974 et le mois d'août 1975. La température varie entre $13{ }^{\circ} \mathrm{C}$ en décembre et $16^{\circ} \mathrm{C}$ en août (Alouf 1977). Ont été récoltés 421 \% a, 110 \% r et 271 ô de G. oronticus (fig. $2 \mathrm{C} 1$ ) et 1 \% a, 2 ㅇ r et 2 ô de $G$. syriacus en février. Le sex-ratio total est de 61 ; les valeurs des six premiers mois de l'année sont inférieures à cette moyenne, le minimum de 37 est atteint en avril et le maximum de 80 en novembre; le passage d'un extrême à un autre est progressif. Le pourcentage de femelles gravides par rapport au total des femelles est toujours supérieur à $60 \%$, le minimum de $64 \%$ s'observe en avril et les maxima de 88 et $89 \%$ en décembre et juillet. La reproduction est donc continue. La taille moyenne des mâles est de 9,25 $\pm 0,19 \mathrm{~mm}$, les classes de 8 et $10 \mathrm{~mm}$ sont plus importantes numériquement que la classe de $9 \mathrm{~mm}$ (fig. 8). La taille moyennne des femelles est de $8,22 \pm 0,14 \mathrm{~mm}$, le minimum de 7,65 $\pm 0,27 \mathrm{~mm}$ s'observe en avril et le maximum de $8,56 \pm 0,15 \mathrm{~mm}$ en août (fig. $3 \mathrm{G}, \mathrm{H}, \mathrm{I}$ ).

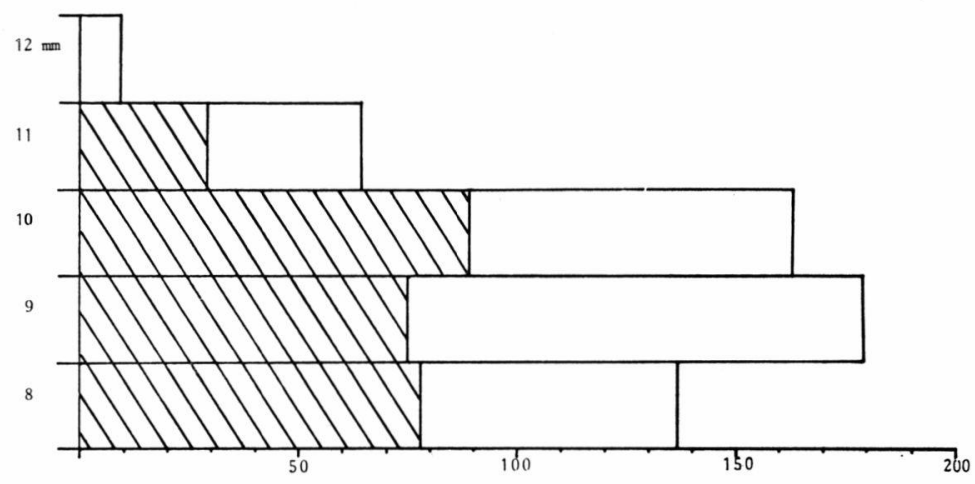

Fig. 8. - Histogramme des mâles de G. oronticus dans les stations $\mathrm{C}_{1}$ (hachures) et $\mathrm{C}_{2}$ (en blanc).

\section{4. - Station C2}

Même emplacement que la station précédente, mais à $2 \mathrm{~m}$ du bord. L'eau, profonde de $30-40 \mathrm{~cm}$, est très rapide. Le fond est formé de pier- 
res et de galets, tapissés de mousse, de $10-25 \mathrm{~cm}$ de diamètre. On ne rencontre que $G$. oronticus, exclusivement, dans cette station.

Les 11 prélèvements, étalés entre le mois d'octobre et le mois d'août, ont donné 844 qa, 168 o r et 552 o (fig. $3 \mathrm{C} 2$ ). Le sex-ratio total est de 62 , les valeurs mensuelles évoluent en dents de scie, elles sont inférieures à cette moyenne au printemps et supérieures en été ; le minimum de 41 s'observe en mai et le maximum de 70 est atteint en juillet. L'activité sexuelle est intense en automne, en hiver et en été, $92 \%$ de femelles gravides en janvier-février et $84 \%$ en juillet. Le ralentissement printanier ne descend pas au-dessous de $40 \%$ en mai. La taille moyenne des mâles est de $9,3 \pm 0,2 \mathrm{~mm}$, celle des femelles $8,42 \pm$ $0,16 \mathrm{~mm}$; le minimum moyen est de $7,76 \pm 0,27 \mathrm{~mm}$ en avril et le maximum moyen $8,76 \pm 0,34 \mathrm{~mm}$ en novembre (fig. $3 \mathrm{~J}, \mathrm{~K}, \mathrm{~L}$ ).

\section{4. - DISCUSSION ET CONCLUSIONS}

Les rythmes de l'activité sexuelle de G. laticoxalis présentent les mêmes évolutions dans l'Oronte et dans les affluents du Litani (Alouf 1979 a). Le pourcentage de femelles sexuellement mûres augmente, ou diminue, en fonction d'un facteur extrinsèque qui est la pluviosité. Mais les données concernant cette espèce, dans l'Oronte, ne sont relatives qu'à une seule station. Aussi la discussion suivante ne concernet-elle que $G$. oronticus.

\section{Activité SEXUELLE}

L'origine de l'évolution de l'activité sexuelle et du sex-ratio de $G$. oronticus dans la station $\mathrm{C}$ ne peut être recherchée dans les variations de la vitesse de l'eau ou de la température, qui sont stables, ni dans la photopériode, vu qu'on observe le déclin de l'activité sexuelle après l'équinoxe du printemps et sa reprise après l'équinoxe de l'automne. Elle semble être, comme pour les Gammares du Litani, en relation avec les précipitations atmosphériques, véhicules de matières nutritives.

L'activité sexuelle atteint deux maxima, en hiver et en été. Le minimum principal est au printemps et le secondaire en automne. Ce cycle rappelle celui des Gammares de l'exsurgence Bardauni dans le bassin du Litani (Alouf 1979 a), avec, toutefois, une différence : le minimum principal dans cette dernière station, s'observe à la fin de l'été et l'autre au printemps. Cette inversion de l'importance relative des minima paraît due à ce que la région du Bardauni reçoit une tranche de $1250 \mathrm{~mm}$ de pluie par an et la saison des pluies tarde jusqu'en mai, tandis que le Hermel ne reçoit qu'une tranche annuelle de 
$240 \mathrm{~mm}$. Le maximum hivernal paraît provoqué par les pluies, et l'estival par l'arrivée des eaux de fonte des neiges (fig. 9).

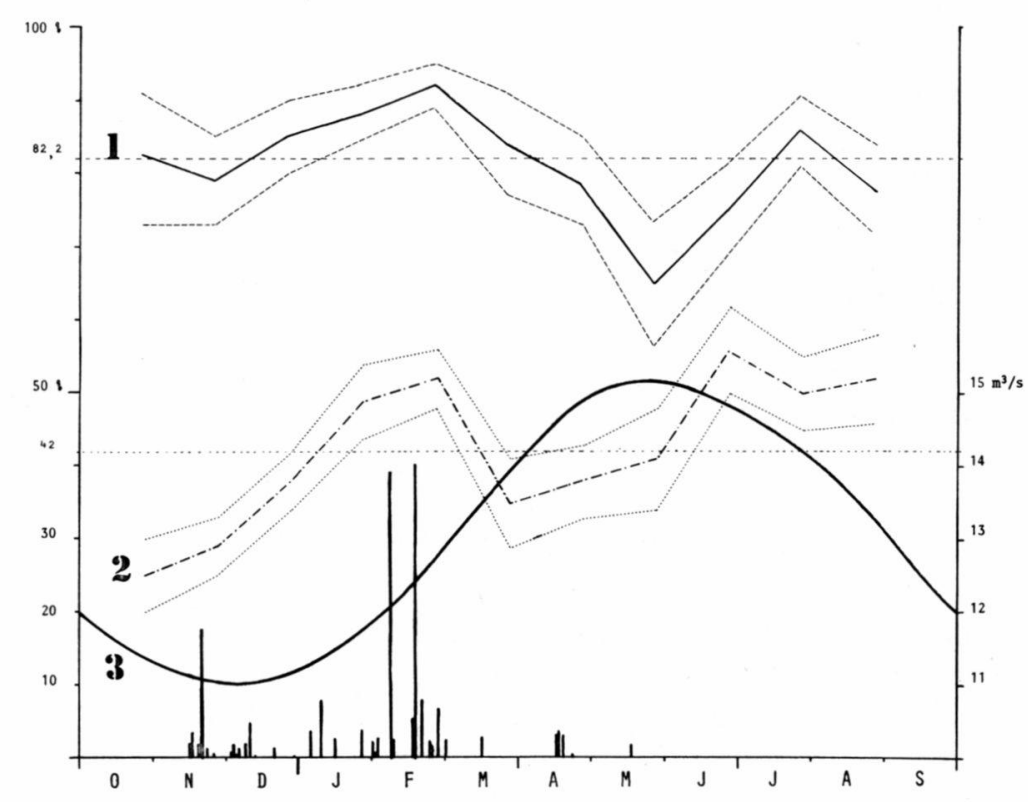

Fig. 9. - Evolution du pourcentage des femelles gravides (1), du sex-ratio (2), débit moyen de l'Oronte pour les périodes 1932-1943 et 1956-1969 (3) et précipitations journalières (traits verticaux).

Les résultats des quatre stations montrent que l'activité sexuelle de G. oronticus présente toujours deux maxima et deux minima. Le minimum printanier moyen est moins prononcé que dans le cours de la rivière, le pourcentage de femelles gravides ( $1518 q$ a et $329 q \mathrm{r}$ ) est de 82. Le sex-ratio, nécessairement bas parce que les femelles sont rares dans la station A, est de l'ordre de 42 ; les deux maxima et les deux minima précèdent ceux de l'activité sexuelle, la corrélation entre le pourcentage des femelles et leur fécondité devient ainsi évidente. Un stimulus, qu'on suppose être l'abondance de matières trophiques, déclenche un processus qui aboutit à une augmentation du nombre de femelles, suivie d'une activité sexuelle intense. La nature de cette corrélation reste à élucider.

Les variations de la taille moyenne et de la densité des adultes, en relation avec l'activité sexuelle, diffèrent de celles du Litani. Elles rappellent celles des Gammares d'Europe. Le nombre d'adultes par prélèvement, mâles et femelles pris ensemble ou à part, passe par un maximum annuel qui varie d'une station à une autre. Il se situe au printemps dans les sources, en été près du bord et en hiver vers le milieu du cours d'eau. 


\section{SeX-Ratio}

Le sex-ratio global montre les mêmes caractéristiques dans les deux bassins. On rencontre ici et là des valeurs élevées, 61, et ailleurs des valeurs moyennes. La seule différence s'observe au niveau de l'exsurgence de 'Ayn-z-Zarqa, où il est très bas, de l'ordre de $4 \%$. Les variations du sex-ratio global nous avaient amené à le considérer comme indice d'optimum écologique (Alouf 1979 a) sans, toutefois, expliquer les mécanismes de contrôle.

Les nouvelles données concernant l'Oronte, stabilité de la température entre autres, permettent de mieux cerner le problème. L'évolution du sex-ratio ne peut être due aux variations de la température, de la photopériode ou de la pluviosité. Elle ne peut l'être qu'à des facteurs intrinsèques, tels qu'une plus grande longévité des femelles comme le suppose Hynes (1955) pour G. pulex : "It seems therefore that in contrast with $G$. duebeni... the males of this species may have a slighty shorter life than the females, although, of course, there remains the possibilit that they are actually born earlier in the season ". Ainsi, en admettant cette hypothèse, les femelles seront plus nombreuses dans les biotopes permanents à conditions écologiques optimales, même si les deux sexes sont à égalité à la naissance.

Cette hypothèse est, toutefois, insuffisante pour expliquer les variations du sex-ratio, surtout si l'on considère le pourcentage d'occupation d'un territoire par rapport aux autres espèces sympatriques comme un indice de son optimum écologique. En combinant cet indice avec l'hypothèse citée plus haut, le sex-ratio minimal de $G$. oronticus devrait être observé dans la station B, ce qui n'est pas le cas. Donc, d'autres facteurs interviennent.

Meijering (1971 et 1972) constata que les mâles des Gammares vivant dans les affluents de la rivière Fulda sont plus agiles et plus rapides à remonter le courant du cours d'eau. Cette agilité des mâles, jointe à la longévité des femelles, expliquent-elles les différences du sex-ratio?

Si l'agilité des Gammares, en général, et des mâles, en praticulier, expliquait leur répartition, comme le suppose Meijering, le sex-ratio devrait être à l'avantage de ces derniers dans les stations $\mathrm{A}$ et $\mathrm{C}$. Or ce n'est pas le cas et d'autres facteurs interviennent donc. En effet, s'il y a abondance de mâles de $G$. oronticus dans la station A comment expliquer l'absence de femelles de $G$. syriacus dans les eaux calmes en amont du barrage? Et pourquoi y a-t-il plus de femelles dans les eaux rapides du cours d'eau, station $\mathrm{C}$, et égalité des sexes dans les eaux relativement calmes, station $B$ ?

Si les femelles sont plus sensibles que les mâles aux variations du milieu, elles sont éliminées les premières lorsque les conditions deviennent défavorables. Cette hypothèse, jointe aux précédentes, fait que le 
sex-ratio devient la résultante de la longévité, de l'agilité et de la tolérance des femelles aux conditions du milieu.

Ainsi, la variation du sex-ratio indique une certaine zonation entre un point sensé être le lieu idéal de l'espèce et les limites qu'elle peut tolérer sans avoir la possibilité de se reproduire. Le lieu idéal ne peut être que le centre du territoire (Mayr 1974). Le sex-ratio s'échelonne entre une valeur optimale permettant un maximum de procréation, avec un minimum d'adultes et la valeur zéro, absence de femelles.

\section{Preferendum écologique}

La proportion de G. oronticus par rapport au total des Gammares dans la station A est de $94,4 \%$. Les récoltes du mois de janvier, des deux côtés de la digue, ont donné soit $G$. syriacus, soit $G$. oronticus exclusivement. Cela laisse penser que la cohabitation entre les deux espèces est accidentelle et qu'il suffit d'un aménagement pour que chacune se retrouve seule. La récolte en amont ayant donné 1 \& a, 5 . $\mathrm{r}$ et 30 ô de $G$. syriacus, celle de l'aval 1 \& a, 3 \& r et 57 o de $G$. oronticus, l'ordre de grandeur et la composition des deux populations sont les mêmes. Ainsi, ces deux espèces réagissent de façon identique aux conditions du milieu. La station A est le lieu de rencontre, ou la frontière, entre deux "territoires", à savoir, le mince filet d'eau de l'amont et le très important cours d'eau engendré par l'exsurgence. Le passage d'un milieu à un autre est brusque; les différences de débit et, par suite, de la vitesse de l'eau, se font sentir de façon notable à cet endroit, ce qui accentue l'instabilité du milieu et augmente les chances de brassage entre les deux espèces. Le sex-ratio est de l'ordre de 4 et la rareté des femelles empêche l'hybridation. Quelle que soit la cause, les possibilités de reproduction, dans cette station, sont minimes. L'équilibre qui s'installe est instable, ce ne sont que des Gammares émigrants qui viennent d'ailleurs vers la périphérie de leur territoire.

Bien que le pourcentage de $G$. oronticus par rapport au total des Gammares de la station B soit de $25,8 \%$, beaucoup moins que dans la station A, que $G$. syriacus soit présent, que $G$. laticoxalis soit prépondérant et que les trois espèces vivent en sympatrie, le sex-ratio de G. oronticus est de $46 \%$. Ce résultat, paradoxal par comparaison avec la première station, ne peut être expliqué que si l'on considère la cohabitation dans la première station comme accidentelle et la sympatrie dans la seconde comme permanente. La relative stabilité des conditions de la station $\mathrm{B}$ rend sa colonisation réelle et effective, par les trois espèces, avec possibilité de se reproduire sur place car le sex-ratio oscille autour de 50. Les variations du débit, qui semblent la cause de la modification des proportions des trois espèces dans cette station, restent dans des limites favorables à G. laticoxalis et font que 
cette forme est dominante toute l'année. La proximité du cours de l'Oronte permet à $G$. oronticus d'envahir cette station lorsque les conditions lui sont plus favorables et les mâles sont les premiers à la coloniser.

En considérant l'élévation du pourcentage d'une espèce comme une inäication d'approche de son optimisme écologique, les conditions de la station B sont favorables, tour à tour, à laticoxalis, oronticus, lati. coxalis puis syriacus. Cette succession paraît en relation avec le cycle annuel des eaux. Les eaux relativement calmes de l'étiage semblent favorables à $G$. syriacus, les eaux vives des crues, au printemps, à $G$. oronticus et les eaux à vitesse moyenne, en automne, en hiver et au début de l'été, à G. laticoxalis.

La présence de 6 individus appartenant à $G$. syriacus à la fin du mois de février, dans la station C1, indiquerait que le bord peut être colonisé par les deux formes. Le sex-ratio de G. oronticus est élevé (61) à peine inférieur à celui observé dans la station C2 (62) où $G$. oronticus se rencontre seul.

De ce qui précède, on peut affirmer que le biotope à eau rapide de l'Oronte, qui a dans sa partie amont un important débit proche de celui du potamon, les eaux rapides d'un rhithron et le régime thermique du crenon (Illies et Botosaneanu 1963) est l'habitat par excellence de G. oronticus. Le biotope de G. laticoxalis, probablement des eaux à vitesse moyenne, reste à délimiter et à préciser.

\section{TRAVAUX CITÉS}

Alouf (N. J.). 1977. - Sur la présence du genre Prosopistoma au Liban. Description de $P$. oronti n. sp. et de $P$. phoenicium n. sp. (Ephemeroptera). Annls Limnol., 13 (2) : 133-139.

Alouf (N. J.). 1979 a. - Cycle de reproduction de deux formes parentes de Gammarus du Liban (Crustacés, Amphipodes). Annls Limnol., 14 (3) : 181-195.

Alouf (N. J.). 1979 b. - Sur la présence du genre Gammarus au Liban, avec description de deux nouveaux taxa (Crustacea, Amphipoda). Bull. Zool. Mus. Univ. Amsterdam, 6 (3) : 177-186.

Berg (K.). 1948. - Biological studies on the river Susaa. Folia Limnol. Scand., 4: $318 \mathrm{p}$.

HYNES (H. B. N.). 1955. - The reproductive cycle of some british freshwater Gammaridae. J. Anim. Ecol., 24 : 352-387.

Illies (J.) \& Botosaneanu (L.). 1963. - Problèmes et méthodes de la classification et de la zonation écologique des eaux courantes, considérées surtout du point de vue faunistique. Mitt. Int. Ver. Limnol., 12 : 1-57.

Karaman (G.S.) \& PinKster (S.). 1977. - Freshwater Gammarus species from Europe, North Africa and adjacent regions of Asia (Crustacea Amphipoda). Part I. Gammarus pulex-group and related species. Bijdr. Dierk., 47 (1) : $1-97$.

MAYR (E.). 1974. - Population, espèces et évolution. Hermann, Paris : 496 p. 
MeIJERING (M. P. D.). 1971. - Die Gammarus-Fauna der Schlitzerländer Fliessgewässer. Arch. Hydrobiol., 68 : 575-608.

MeiJeRING (M. P.D.). 1972. - Experimentelle Untersuchungen zur Drift und Aufwanderung von Gammariden in Fliessgewässern. Arch. Hydrobiol., 70 : 133205. 